\title{
ANALYSIS OF BATCH ARRIVAL QUEUE WITH TWO STAGES OF SERVICE AND PHASE VACATIONS
}

\author{
S. MARAGATHA SUNDARI, S. SRINIVASAN, AND A. RANJITHAM
}

\begin{abstract}
We study a batch arrival queueing system of phase vacation with two stages of service based on a Bernoulli schedule. A single server provides essential service to all arriving customers with service time following a general distribution. After two stages of service completion, the server leaves for phase one vacation of random length with probability $p$ or to continue staying in the system with probability $1-p$. As soon as the completion of phase one vacation, the server undergoes phase two vacation. On completion of two heterogeneous phases of vacation the server returns back to the system. The vacation times are assumed to be general. The server is interrupted and the service interruption follows an exponential distribution. The arrivals follow a Poisson distribution. Using supplementary variable technique, the Laplace transforms of time dependent probabilities of system state are derived. From this we deduce the steady state results. We also obtain the average queue size and average waiting time.
\end{abstract}

\section{INTRODUCTION}

Queueing models with vacation play a major role in manufacturing and production systems, computer and communication systems, service and distribution systems, etc. The studies on queues with batch arrival and vacations have been increased recently. Many real life situations of this model are mostly observed in supermarkets, factories and very large scale manufacturing industries.

Baba [1] studied an $M^{[x]} / \mathrm{G} / 1$ queue with vacation time. Madan and Anabosi [6] studied server vacations based on Bernoulli schedules and a single vacation policy. Madan and Choudhury [8] studied a single server queue with two phases of heterogeneous service under Bernoulli schedule and a general vacation time. Thangaraj and Vanitha [2] studied a single server $\mathrm{M} / \mathrm{G} / 1$ feedback queue with two types of services having general distribution. Madan and Choudhury [7] proposed a queueing system with restricted admissibility of arriving batches. Igaki [12], Levi and Yechilai [13], and Madan and Abu-Davyeh [14] studied vacation queues with different vacation policies.

MISSOURI J. OF MATH. SCI., FALL 2014 


\section{S. MARAGATHA SUNDARI, S. SRINIVASAN, AND A. RANJITHAM}

S. Maragathasundari and S. Srinivasan [15] analyzed the M/G/1 feedback queue with three stages multiple server vacations. S. Maragathasundari and S. Srinivasan [16] analyzed the triple stages of service having compulsory vacation and service interruptions. S. Maragathasundari and S. Srinivasan [17] studied the three phase M/G/1 queue with Bernoulli feedback and multiple server vacation. Various aspects like phase vacations, stages of services, and phases of service have been discussed by $\mathrm{S}$. Maragathasundari and S. Srinivasan [18, 19, 20, 21, 24, 25, 26].

In this paper, we consider non-Markovian single server batch arrival queueing system of two stages of service with interruption, in which we assume that after every service completion, the server has the option to leave for a vacation of random length with probability $p$ or to continue staying in the system with probability $1-p$. The vacation period has two heterogeneous phases. On completion of two vacation phases the server returns back to the system.

This paper is organized as follows. The mathematical description of our model is given in Section 2. Definitions and equations governing the system are given in Section 3. The time dependent solution is obtained in Section 4. The steady state results are derived in Section 5. Average queue size and average waiting time are computed in Sections 6 and 7, respectively. The conclusion is given in Section 8 .

\section{Mathematical Description of the Model}

We assume the following to describe the queueing model of our study.

a) Customers arrive at the system in batches of variable size in a compound Poisson process and are provided one by one service on a first-come-first-served basis. Let $\lambda c_{i} d t$ with $i=1,2, \ldots$ be the first order probability that a batch of $i$ customers arrives at the system during a short interval of time $(t, t+d t)$, where $0 \leq c_{i} \leq 1$, $\sum_{i=1}^{\infty} c_{i}=1$, and $\lambda>0$ is the arrival rate of batches.

b) A single server provides service to all arriving customers with the service time having general distribution. Let $M(\nu)$ and $m(\nu)$ be the distribution and the density function of the service time, respectively.

c) We assume interruptions arrive at random while serving the customers are assumed to occur according to a Poisson process with mean time rate $\alpha>0$. Let $\beta$ be the server's rate of attending interruptions. Furthermore, we assume that once the interruption arrives the customer whose service is interrupted comes back to the head of the queue. Let $\mu(x) d x$ be the conditional probability of completion of service during the interval $(x, x+d x)$ given that 


\section{BATCH ARRIVAL QUEUE WITH TWO STAGES}

the elapsed time is $x$, so that

$$
\mu(x)=\frac{m(x)}{1-M(x)},
$$

and therefore,

$$
m(s)=\mu(s) e^{-\int_{0}^{s} \mu(s) d x} .
$$

d) As soon as the service is over, the server may take a vacation with probability $p$ or may continue staying in the system with probability $1-p$. After phase one vacation completion, the server undergoes phase two vacation. On completion of two heterogeneous phase of vacation the server returns back to the system.

e) The server's vacation time follows a general (arbitrary) distribution with distribution function $V_{i}(t)$ and density function $\nu_{i}(t)$. Let $\gamma_{i}(x) d x$ be the conditional probability of a completion of a vacation during the interval $(x, x+d x)$ given that the elapsed vacation time is $x$, so that

$$
\gamma_{i}(x)=\frac{\nu_{i}(x)}{1-V_{i}(x)} \quad i=1,2,3
$$

and therefore,

$$
\nu_{i}(t)=\gamma_{i}(t) e^{-\int_{0}^{t} \gamma_{i}(s) d x} i=1,2,3 .
$$

f) On returning from vacation the server instantly starts serving the customer at the head of the queue if any.

g) Various stochastic processes involved in the system are assumed to be independent of each other.

\section{Definitions and Equations Governing the System}

We define $P_{n}^{(i)}(x, t)$ to be the probability that at time $t$, the server is providing the $i$ th stage of service and there are $n(n \geq 0)$ customers in the queue excluding the one being served and the elapsed service time for this customer is $x$. Consequently,

$$
P_{n}^{(i)}(t)=\int_{0}^{\infty} P_{n}(x, t) d x
$$

denotes the probability that at time $t$ there are $n$ customers in the queue excluding one customer in the essential service irrespective of the value of $x$ for $i=1,2$.

Let $V_{n}(x, t)$ denote the probability that at time $t$, the server is under vacation with elapsed vacation time $x$ and there are $n(n \geq 0)$ customers 


\section{S. MARAGATHA SUNDARI, S. SRINIVASAN, AND A. RANJITHAM}

in the queue. Consequently,

$$
V_{n}^{(i)}(t)=\int_{0}^{\infty} V_{n}^{(i)}(x, t) d x
$$

denotes the probability that at time $t$ there are $n$ customers in the queue and the server is under vacation irrespective of the value of $x$ for $i=1,2$.

Let $R_{n}(t)$ be the probability that at time $t$, the server is inactive due to arrival of interruption. Let $Q(t)$ be the probability that at time $t$, there are no customers in the queue or in service and the server is idle but available in the system.

According to the mathematical model mentioned above, the system has the following set of differential-difference equations.

$$
\begin{aligned}
\frac{\partial}{\partial x} P_{0}^{(1)}(x, t) & +\frac{\partial}{\partial x} P_{0}^{(1)}(x, t)+\left[\lambda+\alpha+\mu_{1}(x)\right] P_{0}^{(1)}(x, t)=0 \\
\frac{\partial}{\partial x} P_{n}^{(1)}(x, t) & +\frac{\partial}{\partial x} P_{n}^{(1)}(x, t)+\left[\lambda+\alpha+\mu_{1}(x)\right] P_{n}^{(1)}(x, t) \\
& =\lambda \sum_{k=1}^{n} c_{k} P_{n-k}(x, t), \quad n \geq 1 ;
\end{aligned}
$$

$$
\begin{aligned}
\frac{\partial}{\partial x} P_{0}^{(2)}(x, t) & +\frac{\partial}{\partial x} P_{0}^{(2)}(x, t)+\left[\lambda+\alpha+\mu_{2}(x)\right] P_{0}^{(2)}(x, t)=0 \\
\frac{\partial}{\partial x} P_{n}^{(2)}(x, t) & +\frac{\partial}{\partial x} P_{n}^{(2)}(x, t)+\left[\lambda+\alpha+\mu_{2}(x)\right] P_{n}^{(2)}(x, t) \\
& =\lambda \sum_{k=1}^{n} c_{k} P_{n-k}(x, t), \quad n \geq 1
\end{aligned}
$$

$$
\begin{aligned}
\frac{\partial}{\partial x} V_{0}^{(1)}(x, t) & +\frac{\partial}{\partial x} V_{0}^{(1)}(x, t)+\left[\lambda+\alpha+\gamma_{1}(x)\right] V_{0}^{(1)}(x, t)=0 \\
\frac{\partial}{\partial x} V_{n}^{(1)}(x, t) & +\frac{\partial}{\partial x} V_{n}^{(1)}(x, t)+\left[\lambda+\gamma_{1}(x)\right] V_{n}^{(1)}(x, t) \\
& =\lambda \sum_{k=1}^{n} c_{k} V_{n-k}^{(1)}(x, t), \quad n \geq 1
\end{aligned}
$$




$$
\begin{gathered}
\frac{\partial}{\partial x} V_{0}^{(2)}(x, t)+\frac{\partial}{\partial x} V_{0}^{(2)}(x, t)+\left[\lambda+\alpha+\gamma_{1}(x)\right] V_{0}^{(2)}(x, t)=0 \\
\frac{\partial}{\partial x} V_{n}^{(2)}(x, t)+\frac{\partial}{\partial x} V_{n}^{(2)}(x, t)+\left[\lambda+\gamma_{2}(x)\right] V_{n}^{(2)}(x, t) \\
=\lambda \sum_{k=1}^{n} c_{k} V_{n-k}^{(2)}(x, t), \quad n \geq 1 \\
\frac{d}{d t} R_{0}(t)=-(\lambda+\beta) R_{0}(t) \\
\frac{d}{d t} R_{n}(t)=-(\lambda+\beta) R_{n}(t)+\lambda \sum_{k=1}^{n} c_{k} R_{n-k}(t)+\alpha \int_{0}^{\infty} P_{n-1}^{(1)}(x, t) d x \\
+\alpha \int_{0}^{\infty} P_{(n-1)}^{(2)}(x, t) d x ; \text { and } \\
\frac{d}{d t} Q(t)=-\lambda Q(t)+\beta R_{0}(t)+\int_{0}^{\infty} \gamma_{2}(x) V_{0}^{(2)}(x, t) d x \\
+(1-p) \int_{0}^{\infty} \mu(x) P_{0}^{(2)}(x) d x
\end{gathered}
$$

Equations are to be solved subject to the following boundary conditions:

$$
\begin{gathered}
P_{n}^{(1)}(0, t)=\lambda c_{(n+1)} Q(t)+(1-p) \int_{0}^{\infty} \mu_{2}(x) P_{n+1}^{(2)}(x, t) d x \\
\quad+\beta R_{(n+1)}(t)+\int_{0}^{\infty} \gamma_{2}(x) V_{(n+1)}^{(2)}(x, t) d x, \quad n \geq 0 ; \\
P_{n}^{(2)}(0, t)=\int_{0}^{\infty} P_{n}^{(1)}(x, t) \mu_{1}(x) d x ; \\
V_{n}^{(1)}(0, t)=p \int_{0}^{\infty} \mu_{2}(x) P_{n}^{(2)}(x, t) d x, \quad n \geq 0 ; \text { and } \\
V_{n}^{(2)}(0, t)=\int_{0}^{\infty} \gamma_{1}(x) V_{n}^{(1)}(x, t) d x, \quad n \geq 0 .
\end{gathered}
$$

We assume that initially there are no customers in the system and the server is idle. So the initial conditions are

$$
\begin{aligned}
& V_{0}^{(i)}(0)=V_{n}^{(i)}(0)=0, \quad Q(0)=1, \quad R_{n}(0)=0, \\
& P_{n}(0)=0 \text { for } n \geq 0 \text { and } i=1,2 .
\end{aligned}
$$




\section{S. MARAGATHA SUNDARI, S. SRINIVASAN, AND A. RANJITHAM}

\section{Generating Functions of the Queue Length: The Time-Dependent Solution}

In this section, we obtain the transient solution for the above set of differential-difference equations.

Theorem 4.1. The system of differential-difference equations to describe an $M^{[X]} / G / 1$ queue with essential service with service interruption and two phases of vacation are given by equations (1) to (14) with initial conditions (15) and the generating functions of transient solution are given by equation (58a) to (61).

Proof. We define the probability generating functions,

$$
\begin{aligned}
P(x, z, t) & =\sum_{n=0}^{\infty} z^{n} P_{n}^{(i)}(x, t), P^{(i)}(z, t)=\sum_{n=0}^{\infty} z^{n} P_{n}^{(i)}(t) ; i=1,2 \\
R(z, t) & =\sum_{n=0}^{\infty} z^{n} R_{n}(t) ; C(z)=\sum_{n=0}^{\infty} c_{n} z^{n} R_{n}(t) \\
V^{(i)}(x, z, t) & =\sum_{n=0}^{\infty} z^{n} V_{n}^{(i)}(x, t), V^{(i)}(z, t)=\sum_{n=0}^{\infty} z^{n} V_{n}^{(i)}(x, t) \text { for } i=1,2
\end{aligned}
$$

which are convergent inside the circle given by $z \leq 1$ and define the Laplace transform of a function $f(t)$ as

$$
f(s)=\int_{0}^{\infty} e^{-s t} f(t) d t, \quad \Re(s)>0 .
$$

We take the Laplace transform of equations (1) to (14) and using (15), we obtain

$$
\begin{aligned}
& \frac{\partial}{\partial x} \bar{p}_{0}^{(1)}(x, s)+\left[s+\lambda+\alpha+\mu_{1}(x)\right] \bar{p}_{0}^{(1)}(x, s)=0 \\
& \frac{\partial}{\partial x} \bar{p}_{n}^{(1)}(x, s)+\left[s+\lambda+\alpha+\mu_{1}(x)\right] \bar{p}_{n}^{(1)}(x, s)=\lambda \sum_{k=1}^{n} c_{k} \bar{P}_{n-k}(x, s), n \geq 1 \\
& \frac{\partial}{\partial x} \bar{p}_{0}^{(2)}(x, s)+\left[s+\lambda+\alpha+\mu_{2}(x)\right] \bar{p}_{0}^{(2)}(x, s)=0 ; \\
& \frac{\partial}{\partial x} \bar{p}_{n}^{(2)}(x, s)+\left[s+\lambda+\alpha+\mu_{2}(x)\right] \bar{p}_{n}^{(2)}(x, s)=\lambda \sum_{k=1}^{n} c_{k} \bar{P}_{n-k}(x, s), n \geq 1
\end{aligned}
$$




$$
\begin{gathered}
\frac{\partial}{\partial x} \bar{V}_{0}^{(1)}(x, s)+\left[s+\lambda+\gamma_{1}(x)\right] \bar{V}_{0}^{(1)}(x, s)=0 \\
\frac{\partial}{\partial x} \bar{V}_{n}^{(1)}(x, s)+\left[s+\lambda+\gamma_{1}(x)\right] \bar{V}_{n}^{(1)}(x, s)=\lambda \sum_{k=1}^{n} c_{k} \bar{V}_{n-k}^{(1)}(x, s), n \geq 1 ; \\
\frac{\partial}{\partial x} \bar{V}_{0}^{(2)}(x, s)+\left[s+\lambda+\gamma_{2}(x)\right] \bar{V}_{0}^{(2)}(x, s)=0 ; \\
\frac{\partial}{\partial x} \bar{V}_{n}^{(2)}(x, s)+\left[s+\lambda+\gamma_{2}(x)\right] \bar{V}_{n}^{(2)}(x, s)=\lambda \sum_{k=1}^{n} C_{K} \bar{V}_{n-k}^{(2)}(x, s), n \geq 1 \\
(s+\lambda+\beta) \bar{R}_{0}(s) 7=0 ; \\
(s+\lambda+\beta) \bar{R}_{n}(s)=\lambda \sum_{n=0}^{\infty} C_{K} \bar{R}_{n-k}(s)+\left[\alpha \int_{0}^{\infty} \bar{P}_{n-1}^{(1)}(x, s) d x\right. \\
\left.+\alpha \int_{0}^{\infty} \bar{P}_{n-1}^{(2)}(x, s) d x\right], n \geq 1 \\
(s+\lambda) \bar{Q}(s)=1+\beta \bar{R}_{0}(s)+\int_{0}^{\infty} \gamma_{2}(x) \bar{V}_{0}^{(2)}(x, s) d x \\
+(1-p) \int_{0}^{\infty} \mu(x) \bar{P}_{0}^{(2)}(x, s) d x ;
\end{gathered}
$$

$$
\begin{aligned}
\bar{P}_{n}^{(1)}(0, s)= & \lambda c_{n+1} \bar{Q}(S)+\beta \bar{R}_{n+1}(s)+(1-p) \int_{0}^{\infty} \mu_{2}(x) \bar{P}_{n+1}^{(2)}(x, s) d x \\
& +\int_{0}^{\infty} \gamma_{2}(x) \bar{V}_{n+1}^{(2)}(x, s) d x
\end{aligned}
$$$$
\bar{P}_{n}^{(2)}(0, s)=\int_{0}^{\infty} \bar{P}_{n}^{(1)}(x, s) \mu_{1}(x) d x
$$$$
\bar{V}_{n}^{(1)}(0, s)=p \int_{0}^{\infty} \bar{P}_{n}^{(2)}(x, s) \mu_{2}(x) d x, n \geq 0 ; \quad \text { and }
$$$$
\bar{V}_{n}^{(2)}(0, s)=\int_{0}^{\infty} \bar{V}_{n}^{(1)}(x, s) \gamma_{1}(x) d x, n \geq 0 .
$$

Now multiplying equations (21), (21b), (23), (25) by $z^{n}$ and summing over $n$ from 0 to $\infty$, adding equations (20), (21a), (22), (24) and using the 
S. MARAGATHA SUNDARI, S. SRINIVASAN, AND A. RANJITHAM

generating functions defined in equations (17) and (18) we obtain

$$
\begin{gathered}
\frac{\partial}{\partial_{x}} \bar{P}_{n}^{(1)}(x, z, s)+\left[s+\lambda-\lambda C(z)+\alpha+\mu_{1}(x)\right] \bar{P}_{n}^{(1)}(x, z, s)=0 \\
\frac{\partial}{\partial x} \bar{P}_{n}^{(2)}(x, z, s)+\left[s+\lambda-\lambda C(z)+\alpha+\mu_{2}(x)\right] \bar{P}_{n}^{(2)}(x, z, s)=0 ; \\
\frac{\partial}{\partial_{x}} \bar{V}_{n}^{(1)}(x, z, s)+\left[s+\lambda-\lambda C(z)+\gamma_{1}(x)\right] \bar{V}^{(1)}(x, z, s)=0 ; \\
\frac{\partial}{\partial_{x}} \bar{V}_{n}^{(2)}(x, z, s)+\left[s+\lambda-\lambda C(z)+\gamma_{2}(x)\right] \bar{V}^{(2)}(x, z, s)=0 ; \text { and } \\
(s+\lambda-\lambda C(z)+\beta) \bar{R}(z, s)=\alpha z\left[\int_{0}^{\infty} \bar{P}^{(1)}(x, z, s) d x\right. \\
\left.+\int_{0}^{\infty} \bar{P}^{(2)}(x, z, s) d x\right] .
\end{gathered}
$$

For the boundary conditions, we multiply both sides of equation (29a) by $z^{n}$, sum over $n$ from 0 to $\infty$, and use the equations (17) and (18) to get

$$
\begin{aligned}
z \bar{P}^{1}(0, z, s)= & \lambda C(Z) \bar{Q}(S)+\beta \bar{R}(z, s)-\beta \bar{R}_{0}(s) \\
& +(1-p) \int_{0}^{\infty} \mu_{2}(x) \bar{P}^{(2)}(x, z, s) d x \\
& -(1-p) \int_{0}^{\infty} \mu_{0}(x) \bar{P}^{(2)}(x, z, s) d x \\
& +\int_{0}^{\infty} \gamma_{2}(x) \bar{V}^{(2)}(x, z, s) d x \\
& -\int_{0}^{\infty} \gamma_{2}(x) \bar{V}_{0}^{(2)}(x, z, s) d x .
\end{aligned}
$$

Using equation (28), equation (36) becomes

$$
\begin{aligned}
z \bar{P}^{(1)}(0, z, s)= & {[1-s \bar{Q}(s)]+\lambda(C(z)-1) \bar{Q}(S)+\beta \bar{R}(z, s) } \\
& +\int_{0}^{\infty} \gamma_{2}(x) \bar{V}^{(2)}(x, z, s) d x \\
& +(1-p) \int_{0}^{\infty} \mu_{2}(x) \bar{P}^{(2)}(x, z, s) d x ; \text { and }
\end{aligned}
$$

$\bar{P}^{(2)}(0, z, s)=\int_{0}^{\infty} \bar{P}^{(1)}(x, z, s) \mu_{2}(x) d x$. 


\section{BATCH ARRIVAL QUEUE WITH TWO STAGES}

Performing a similar operation on equations (30) to (31), we obtain

$$
\begin{gathered}
\bar{V}^{(1)}(0, z, s)=p \int_{0}^{\infty} \mu_{2}(x) \bar{P}^{(2)}(x, z, s) d x ; \text { and } \\
\bar{V}^{(2)}(0, z, s)=\int_{0}^{\infty} \gamma_{1}(x) \bar{V}^{(1)}(x, z, s) d x .
\end{gathered}
$$

Integrating equation (32a) between 0 to $x$, we get

$$
\bar{P}^{(1)}(x, z, s)=\bar{P}^{(1)}(0, z, s) e^{-[s+\lambda-\lambda C(z)+\alpha] x-\int_{0}^{\infty} \mu_{1}(t) d t} .
$$

Integrating equation $(32 \mathrm{~b})$ between 0 to $x$, we get

$$
\bar{P}^{(2)}(x, z, s)=\bar{P}^{(2)}(0, z, s) e^{-[s+\lambda-\lambda C(z)+\alpha] x-\int_{0}^{\infty} \mu_{2}(t) d t}
$$

where $\bar{P}^{(1)}(0, z, s)$ is given by equation (37). Again integrating equation (40a) by parts with respect to $x$ yields

$$
\bar{P}^{(1)}(z, s)=\bar{P}^{(1)}(0, z, s)\left\{\frac{1-\bar{M}_{1}(s+\lambda-\lambda C(z)+\alpha)}{s+\lambda-\lambda C(z)+\alpha}\right\}
$$

where $\bar{P}^{(2)}(0, z, s)$ is given by equation (37a). Again integrating equation (40b) by parts with respect to $x$ yields

$$
\bar{P}^{(2)}(z, s)=\bar{P}^{(2)}(0, z, s)\left\{\frac{1-M_{2}(s+\lambda-\lambda C(z)+\alpha)}{s+\lambda-\lambda C(z)+\alpha}\right\},
$$

where

$$
\bar{M}_{1}(s+\lambda-\lambda C(z)+\alpha)=\int_{0}^{\infty} e^{-[s+\lambda-\lambda C(z)+\alpha] x} d M_{1}(x)
$$

and

$$
\bar{M}_{2}(s+\lambda-\lambda C(z)+\alpha)=\int_{0}^{\infty} e^{-[s+\lambda-\lambda C(z)+\alpha] x} d M_{2}(x)
$$

are the Laplace-Stieltjes transform of the first stage of service time $M_{1}(x)$ and second stage of service time $M_{2}(x)$. Now multiplying both sides of equation (40a) by $\mu_{1}(x)$ and integrating over $x$ we obtain

$$
\int_{0}^{\infty} \bar{P}^{(1)}(x, z, s) \mu_{1}(x) d x=\bar{P}^{(1)}(0, z, s) \bar{M}_{1}(s+\lambda-\lambda C(z)+\alpha)
$$

MISSOURI J. OF MATH. SCI., FALL 2014 


\section{S. MARAGATHA SUNDARI, S. SRINIVASAN, AND A. RANJITHAM}

which is the Laplace-Stieltjes transform of the second stage of service time $B_{2}(x)$. Now multiplying both sides of equation (40b) by $\mu_{2}(x)$ and integrating over $x$ we obtain

$$
\int_{0}^{\infty} \bar{P}^{(2)}(x, z, s) \mu_{2}(x) d x=\bar{P}^{(2)}(0, z, s) \bar{M}_{2}(s+\lambda-\lambda C(z)+\alpha) .
$$

Similarly, on integrating equations (33) to (34) from 0 to $x$,

$$
\bar{V}^{(1)}(x, z, s)=\bar{V}^{(1)}(0, z, s) e^{-[s+\lambda-\lambda C(z)] x-\int_{0}^{\infty} \gamma_{1}(t) d t}
$$

and

$$
\bar{V}^{(2)}(x, z, s)=\bar{V}^{(2)}(0, z, s) e^{-[s+\lambda-\lambda C(z)] x-\int_{0}^{\infty} \gamma_{2}(t) d t}
$$

where $\bar{V}^{(1)}(0, z, s)$ and $\bar{V}^{(2)}(0, z, s)$ are given by equations (38) and (39). Again integrating equations (44) and (45) by parts with respect to $x$ yields,

$$
\begin{aligned}
& \bar{V}^{(1)}(z, s)=\bar{V}^{(1)}(0, z, s)\left\{\frac{1-\bar{V}_{1}(s+\lambda-\lambda C(z))}{s+\lambda-\lambda C(z)}\right\} ; \\
& \bar{V}^{(2)}(z, s)=\bar{V}^{(2)}(0, z, s)\left\{\frac{1-\bar{V}_{2}(s+\lambda-\lambda C(z))}{s+\lambda-\lambda C(z)}\right\} ;
\end{aligned}
$$

where

$$
\begin{aligned}
& \bar{V}_{1}(s+\lambda-\lambda C(z))=\int_{0}^{\infty} e^{-[s+\lambda-\lambda C(z)+\alpha] x} d V_{1}(x) ; \\
& \bar{V}_{2}(s+\lambda-\lambda C(z))=\int_{0}^{\infty} e^{-[s+\lambda-\lambda C(z)+\alpha] x} d V_{2}(x)
\end{aligned}
$$

is the Laplace-Stieltjes transform of the first phase and second phase of vacation $V_{1}(x)$ and $V_{2}(x)$, respectively. Now multiplying both sides of equations (44) and (45) by $\gamma_{1}(x)$ and $\gamma_{2}(x)$ and integrating over $x$ we obtain

$$
\begin{aligned}
& \int_{0}^{\infty} \bar{V}^{(1)}(x, z, s) \gamma_{1}(x) d x=\bar{V}^{(1)}(0, z, s) \bar{V}^{(1)}\left[\left(f_{3}(Z)\right)\right] ; \\
& \int_{0}^{\infty} \bar{V}^{(2)}(x, z, s) \gamma_{2}(x) d x=\bar{V}^{(2)}(0, z, s) \bar{V}^{(2)}\left[\left(f_{3}(Z)\right)\right] .
\end{aligned}
$$

Using equation (43a) and (43b) in equation (38), we obtain

$$
\bar{V}^{(1)}(0, z, s)=p \bar{M}_{1}\left(f_{1}(z)\right) \bar{p}^{(2)}(0, z, s) .
$$

Now using equation (50) and (52) in (39), we obtain

$$
\bar{V}^{(2)}(0, z, s)=p \bar{V}_{1}\left(f_{3}(z)\right) \bar{M}_{2}\left(f_{1}(z)\right) \bar{p}^{(2)}(0, z, s) .
$$

From (35), (40a), and (40b), we obtain

$$
\bar{R}(z, s)=\frac{a z}{\left(f_{1}(z)\right)\left(f_{2}(z)\right)} \bar{p}^{(1)}(0, z, s)\left[1-\bar{M}_{1}\left(f_{1}(Z)\right) \bar{M}_{2}\left(f_{1}(z)\right)\right] .
$$




\section{BATCH ARRIVAL QUEUE WITH TWO STAGES}

Now using equation (43b), (51), and (54), we obtain

$$
\begin{gathered}
\bar{p}^{(1)}(0, z, s)=\frac{[1-s \bar{Q}(s)+\lambda(C(z)-1) \bar{Q}(s)] f_{1}(z) f_{2}(z)}{D R} \text { and }(55 \mathrm{a}) \\
\bar{p}^{(2)}(0, z, s)=\frac{[1-s \bar{Q}(s)+\lambda(C(z)-1) \bar{Q}(s)] f_{1}(z) f_{2}(z) \bar{M}_{1}\left(f_{1}(z)\right)}{D R} .
\end{gathered}
$$

Similarly using equation (66), in equations (61), (62), and (63), we obtain

$$
\begin{array}{r}
\bar{V}^{(1)}(0, z, s)=p\left\{\bar{p}^{(1)}(0, z, s)\right\} \bar{M}_{1}\left(f_{1}(z)\right) \bar{M}_{2}\left(f_{1}(z)\right), \\
\bar{V}^{(2)}(0, z, s)=p \bar{V}^{(1)}\left(f_{3}(z)\right) \bar{M}_{1}\left(f_{1}(z)\right) \bar{p}^{(1)}(0, z, s) \bar{M}_{2}\left(f_{1}(z)\right),
\end{array}
$$

where

$$
\begin{aligned}
D R= & \left\{\begin{array}{c}
z-p \bar{V}_{1}\left(f_{3}(z)\right) \bar{V}_{2}\left(f_{3}(z)\right) \\
\bar{M}_{1}\left(f_{1}(z)\right) \bar{M}_{2}\left(f_{1}(z)\right) \\
-(1-p) \bar{M}_{1}\left(f_{1}(z)\right) \bar{M}_{2}\left(f_{1}(z)\right)
\end{array}\right\} f_{1}(z) f_{2}(z) \\
& -\beta \alpha z\left(1-\bar{M}_{1}\left(f_{1}(z)\right) \bar{M}_{2}\left(f_{1}(z)\right) \times\left\{\bar{M}_{1}\left(f_{1}(z)\right)\left(1-\bar{M}_{2}\left(f_{1}(z)\right)\right\}\right.\right.
\end{aligned}
$$

where $s+\lambda-\lambda C(z)+\alpha=f_{1}(z), s+\lambda-\lambda C(z)+\beta=f_{2}(z), \bar{V}_{1}(s+\lambda-\lambda C(z))=$ $\bar{V}\left(f_{3}(z)\right)$. Using equations (55a) to (56) in equations (41a), (41b), (46), and (47), we obtain

$$
\bar{p}^{(1)}(z, s)=\frac{f_{2}(z)\left\{(1-s \bar{Q}(s)+\lambda(C(z)-1) \bar{Q}(s)\}\left\{1-\bar{M}_{1}\left(f_{1}(z)\right)\right\}\right.}{D R},
$$

$$
\begin{aligned}
\bar{p}^{(2)}(z, s)= & \frac{\left\{(1-s \bar{Q}(s)+\lambda(C(z)-1) \bar{Q}(s)\} f_{1}(z)\right.}{D R} \\
& \times\left\{\begin{array}{c}
\bar{M}_{1}\left(f_{1}(z)\right) \\
\left\{1-\bar{M}_{1}\left(f_{1}(z)\right)\right\}
\end{array}\right\} .
\end{aligned}
$$

$$
\begin{aligned}
\bar{V}^{(1)}(z, s) & =\frac{\{(1-s \bar{Q}(s)+\lambda(C)(z)-1) \bar{Q}(s)\}}{D R} p \bar{M}_{1}\left(f_{1}(z)\right) \\
& \times \bar{M}_{2}\left(f_{1}(z)\right) f_{1}(z) f_{2}(Z)\left\{\frac{1-\bar{V}_{1}\left(f_{3}(z)\right)}{s+\lambda-\lambda C(z)}\right\}
\end{aligned}
$$

MISSOURI J. OF MATH. SCI., FALL 2014 


$$
\begin{gathered}
\bar{V}^{(2)}(z, s)=\frac{\{(1-s \bar{Q}(s)+\lambda(C(z)-1) \bar{Q}(s)\}}{D R} p \bar{M}_{1}\left(f_{1}(z)\right) \\
\times \bar{M}_{2}\left(f_{1}(z)\right) \bar{V} f_{1}(z) f_{2}(Z)\left\{\frac{1-\bar{V}_{2}\left(f_{3}(z)\right)}{s+\lambda-\lambda C(z)}\right\} \\
\bar{R}(z, s)= \\
\times\left[1-\bar{M}_{1}\left(f_{1}(z)\right) \bar{M}_{2}\left(f_{1}(z)\right)\right]
\end{gathered}
$$

where DR is given by equation (57). Thus, $\bar{P}^{(1)}(z, s), \bar{P}^{(2)}(z, s), \bar{V}^{(1)}(z, s)$, $\bar{V}^{(2)}(z, s)$, and $\bar{R}(z, s)$ are completely determined from equations (58a) to (61) which complete the proof of the theorem.

\section{The Steady State Results}

In this section, we shall derive the steady state probability distribution for our queueing model. These probabilities are obtained by suppressing the argument $t$ whereever it appears in the time dependent analysis. This can be obtained by applying the well-known Tauberian property

$$
\lim _{s \rightarrow 0} s \bar{f}(s)=\lim _{t \rightarrow \infty} f(t) .
$$

In order to determine $\bar{P}^{(1)}(z, s), \bar{P}^{(2)}(z, s), \bar{V}^{(1)}(z, s), \bar{V}^{(2)}(z, s)$, and $\bar{R}(z, s)$ completely, we have yet to determine the unknown $Q$ which appears in the numerators of the right-hand sides of equations (58a) to (61). For that purpose, we shall use the normalizing condition

$$
P_{(1)}^{(1)}+P_{(1)}^{(2)}+\bar{V}^{(1)}(1)+\bar{V}^{(2)}(1)+R(1)+Q=1 .
$$

Theorem 5.1. The steady state probabilities for an $M^{[x]} / G / 1$ queue of two stages of service with service interruption and two phases vacation are given by

$$
\begin{aligned}
p_{(1)}^{(1)} & =\frac{\beta \lambda E(I)\left\{1-\bar{M}_{1}(\alpha)\right\} Q}{d r}, \\
p_{(1)}^{(2)} & =\frac{\beta \lambda \bar{M}_{1}(\alpha) E(I)\left\{1-\bar{M}_{2}(\alpha)\right\} Q}{d r}
\end{aligned}
$$




$$
\begin{aligned}
\bar{V}^{(1)}(1) & =\frac{\lambda p \alpha \beta E(I) \bar{M}_{1}(\alpha) \bar{M}_{2}(\alpha) E\left(V_{1}\right) Q}{d r}, \\
\bar{V}^{(2)}(1) & =\frac{\lambda p \alpha \beta E(I) \bar{M}_{1}(\alpha) \bar{M}_{2}(\alpha) E\left(V_{2}\right) Q}{d r}, \\
R(I) & =\frac{\lambda \alpha E(I)\left\{1-\bar{M}_{1}(\alpha) \bar{M}_{2}(\alpha)\right\} Q}{d r},
\end{aligned}
$$

where

$d r=-\lambda(\alpha+\beta)\left[1-\bar{M}_{1}(\alpha) \bar{M}_{2}(\alpha)\right]+\alpha \beta\left[\bar{M}_{1} \bar{M}_{2}\left(1-p \lambda E(I)\left(E\left(v_{1}\right)+E\left(v_{2}\right)\right)\right)\right]$.

$E(V)=E\left(V_{1}\right)+E\left(V_{2}\right), P(1), \bar{V}^{(1)}(1), \bar{V}^{(2)}(1), R(1)$ and $Q$ are the steady state probabilities that the server is providing essential service, first phase of vacation, second phase of vacation and server under idle, respectively without regard to the number of customers in the queue.

Proof. Multiplying both sides of equations (58a) to (61) by $s$, taking limit $s \longrightarrow 0$, applying Tauberian property and simplifying, we obtain

$$
\begin{aligned}
p^{(1)}(z)= & \frac{f_{2}(z)\left(1-\bar{M}_{1}\left(f_{1}(z)\right)\right) \lambda(C(Z)-1) Q}{d r} \\
p^{(2)}(z)= & \frac{f_{2}(z) \bar{M}_{1}\left(f_{1}(z)\right)\left(1-\bar{M}_{2}\left(f_{1}(z)\right)\right) \lambda(C(z)-1) Q}{d r} \\
V^{(1)}(z)= & \frac{p f_{1}(z) f_{2}(z) \bar{M}_{1}\left(f_{1}(z)\right) \bar{M}_{2}\left(f_{1}(z)\right)\left(\bar{V}_{1}(\lambda-\lambda C(z)-1) Q\right.}{d r}, \\
V^{(2)}(z)= & \frac{Q p f_{1}(z) f_{2}(z) \bar{V}_{1}\left(f_{3}(z)\right) \bar{M}_{1}\left(f_{1}(z)\right) \bar{M}_{2}\left(f_{1}(z)\right)}{d r} \\
& \times\left(\bar{V}_{2}(\lambda-\lambda C(z)-1)-1\right)
\end{aligned}
$$

and

$$
R(z)=\frac{a z\left(1-\bar{M}_{1}\left(f_{1}(z)\right) \bar{M}_{2}\left(f_{1}(z)\right)\right) \lambda(C(z)-1) Q}{d r} .
$$

Let $W_{q}(z)$ denote the probability generating function of the queue size irrespective of the state of the system. Then adding equations (69) to (73) we obtain

$$
W_{q}(z)=p^{(1)}(z)+P^{(2)}(z)+V^{(1)}(z)+V^{(2)}(z)+R(z) \text {, and hence }
$$




$$
\begin{aligned}
W_{q}(z)= & \frac{f_{2}(z)\left(1-\bar{M}_{1}\left(f_{1}(z)\right)\right) \lambda(C(z)-1) Q}{d r} \\
& +\frac{f_{2}(z) \bar{M}_{1}\left(f_{1}(z)\right)\left(1-\bar{M}_{2}\left(f_{1}(z)\right) \lambda(C(z)-1) Q\right.}{d r} \\
& +\frac{p f_{1}(z) f_{2}(z) \bar{M}_{1}\left(f_{1}(z)\right) \bar{M}_{2}\left(f_{1}(z)\right)\left(\bar{V}_{1}(\lambda-\lambda C(z)-1)\right) Q}{d r} \\
& +\frac{Q p f_{1}(z) f_{2}(z) \bar{V}_{1}\left(f_{3}(z)\right) \bar{M}_{1}\left(f_{1}(z)\right) \bar{M}_{2}\left(f_{1}(z)\right)}{d r} \\
& \times\left(\bar{V}_{2}(\lambda-\lambda C(z)-1)-1\right) \\
& +\frac{a z\left(1-\bar{M}_{1}\left(f_{1}(z)\right) \bar{M}_{2}\left(f_{1}(z)\right)\right) \lambda(C(z)-1) Q}{d r} .
\end{aligned}
$$

We see that for $z=1, W_{Q}(1)$ is the indeterminate of the form $0 / 0$. Therefore, we apply L'Hopital's rule and on simplifying we obtain the result (89), where $C(1)=1, C^{\prime}(1)=E(I)$ is the mean batch size of the arriving customers. $-\bar{V}_{i}^{\prime}(0)=E\left(V_{i}\right), i=1,2$.

$$
\begin{aligned}
W_{q}(1)= & {\left[\lambda Q \left\{(\alpha+\beta)(E(I))\left[1-\bar{M}_{1}(\alpha) \bar{M}_{2}(\alpha)\right]\right.\right.} \\
& \left.\left.+\alpha \beta\left[\bar{M}_{1}(\alpha) \bar{M}_{2}(\alpha)\left(1-p \lambda E(I)\left(E\left(v_{1}\right)+E\left(v_{2}\right)\right)\right)\right]\right\}\right] \\
& {\left[-\lambda(\alpha+\beta)\left[1-\bar{M}_{1}(\alpha) \bar{M}_{2}(\alpha)\right]\right.} \\
& \left.+\alpha \beta\left[\bar{M}_{1}(\alpha) \bar{M}_{2}(\alpha)\left(1-p \lambda E(I)\left(E\left(v_{1}\right)+E\left(v_{2}\right)\right)\right)\right]\right]^{-1}
\end{aligned}
$$

And $d r$ is given by equation (68). Therefore, adding $Q$ to equation (75), equating to 1 and simplifying, we obtain

$$
Q=1-\rho
$$

and hence the utilization factor $\rho$ of the system is given by

$$
\begin{aligned}
\rho= & {\left[\lambda Q \left\{(\alpha+\beta)(E(I))\left[1-\bar{M}_{1}(\alpha) \bar{M}_{2}(\alpha)\right]\right.\right.} \\
& \left.\left.+\alpha \beta\left[\bar{M}_{1}(\alpha) \bar{M}_{2}(\alpha)\left(1-p \lambda E(I)\left(E\left(v_{1}\right)+E\left(v_{2}\right)\right)\right)\right]\right\}\right] \\
& {\left[-\lambda(\alpha+\beta)\left[1-\bar{M}_{1}(\alpha) \bar{M}_{2}(\alpha)\right]\right.} \\
& \left.+\alpha \beta\left[\bar{M}_{1}(\alpha) \bar{M}_{2}(\alpha)\left(1-p \lambda E(I)\left(E\left(v_{1}\right)+E\left(v_{2}\right)\right)\right)\right]\right]^{-1}
\end{aligned}
$$

where $\rho<1$ is the stability condition under which the steady state exists. Equation (76) gives the probability that the server is idle. Substituting $Q$ 


\section{BATCH ARRIVAL QUEUE WITH TWO STAGES}

from (76) into (74), we have completely and explicitly determined $W_{q}(z)$, the probability generating function of the queue size.

\section{The Average Queue Size}

Let $L_{q}$ denote the average number of customers in the queue under the steady state. Then

$$
L_{q}=\frac{d}{d z} W_{q}(z) \text { at } Z=1 .
$$

Since this formula gives $0 / 0$ form, then we write $W_{q}(z)$ given in (74) as $W_{q}(z)=\frac{N(z)}{D(z)}$ where $N(z)$ and $D(z)$ are numerator and denominator of the right-hand side of (74), respectively. Then we use

$$
L_{q}=\lim _{z \rightarrow 1} \frac{d}{d z} W_{q}(z)=\lim _{z \rightarrow 1}\left(\frac{D^{\prime}(1) N^{\prime \prime}(1)-N^{\prime}(1) D^{\prime \prime}(1)}{2\left(D^{\prime}(1)\right)^{2}}\right) Q
$$

where primes and double primes in (78) denote first and second derivative at $z=1$, respectively. Carrying out the derivative at $z=1$ we have

$$
\begin{aligned}
N^{\prime}(1)= & \lambda Q\left\{(\alpha+\beta)(E(I))\left[1-\bar{M}_{1}(\alpha) \bar{M}_{2}(\alpha)\right]\right. \\
& \left.+\alpha \beta \bar{M}_{1}(\alpha) \bar{M}_{2}(\alpha) E(I)\left[E\left(v_{1}\right)+E\left(v_{2}\right)\right]\right\} . \\
D^{\prime}(1)=- & \lambda(\alpha+\beta)\left[1-\bar{M}_{1}(\alpha) \bar{M}_{2}(\alpha)\right] \\
& +\alpha \beta\left[\bar{M}_{1} \bar{M}_{2}\left(1-p \lambda E(1)\left(E\left(v_{1}\right)+E\left(v_{2}\right)\right)\right)\right] .
\end{aligned}
$$

Similarly, the second order derivatives are calculated. Substituting all the derivatives in equation (78) we obtain $L_{q}$ in the closed form. Furthermore, we find the mean system size $L$ using Little's formula. Thus, we have

$$
L=L_{q}+\rho
$$

where $L_{q}$ has been found by equation (78) and $\rho$ is obtained from equation (77).

\section{The Average Waiting Time}

Let $W_{q}$ and $W$ denote the mean waiting time in the queue and in the system, respectively. Then using Little's formula, we obtain

$$
W_{q}=\frac{L_{q}}{\lambda}
$$

and

$$
W=\frac{L}{\lambda}
$$

where $L_{q}$ and $L$ have been found in equations (78) and (81).

MISSOURI J. OF MATH. SCI., FALL 2014 


\section{S. MARAGATHA SUNDARI, S. SRINIVASAN, AND A. RANJITHAM}

\section{Conclusion}

In this paper, we have studied a batch arrival, two stage heterogeneous services with random breakdown, and Bernoulli schedule with two phases of server vacation. This paper clearly analyzes the time dependent solution and steady state results, and some performance measures of the queueing system. The result of this paper is useful for computer communication network, and large scale industrial production lines.

\section{REFERENCES}

[1] Y. Baba, On the $M^{[x]} / G / 1$ queue with vacation time, Operations Research Letters, 5 (1986), 93-98.

[2] G. Chodhury, An $M^{[x]} / G / 1$ queueing system with a set up period and a vacation period, Questa, 36 (2000), 23-28.

[3] M. Cramer, Stationary distributions in a queueing system with a vacation times and limited service, Queueing Systems, 4 (1989), 57-68.

[4] N. Igaki, Exponential two server queue with $N$-policy and general vacation, Queueing Systems, 10 (1992), 279-294.

[5] B. Krishnakumar, A. Vijayakumar, and D. Arividainambi, An $M / G / 1$ retrial queueing system with two phase service and preemptive resume, Annals of Operations Research, 113 (2002), 61-79.

[6] Y. Levi and U. Yechilai, An $M / M / s$ queue with server vacations, Infor., 14 (1976), $153-163$.

[7] K. C. Madan, On a single server with two stage heterogeneous service and binomial schedule server vacations, Egyptian Statistical Journal, 40.1 (2000), 39-55.

[8] K. C. Madan and R. F. Anabosi, A single server queue with two types of service, Bernoulli schedule server vacations and a single vacation policy, Pakistan Journal of Statistics, 19 (2003), 331-442.

[9] K. C. Madan and G. Chodhury, An $M^{[x]} / G / 1$ queue with Bernoulli vacation schedule under restricted admissibility policy, Sankhaya, 66 (2004), 172-193.

[10] K. C. Madan and G. Chodhury, A two stage arrival queueing system with a modified Bernoulli schedule vacation under N-policy, Mathematical and Computer Modeling, 42 (2005), 71-85.

[11] K. C. Madan, F. A. Maraghi, and K. D. Dowman, Batch arrival queueing system with random breakdowns and Bernoulli schedule server vacations having general vacation time distribution, Information and Management Sciences, 20 (2009), 5570 .

[12] K. C. Madan, An $M / G / 1$ queue with second optional service, Queueing Systems, 34 (2000), 37-46.

[13] K. C. Madan and A. Z. Abu-Dayyeh, On a single server queue with optional phase type server vacations based on exhaustive deterministic service and a single vacation policy, Applied Mathematics and Computation, 149 (2004), 723-734.

[14] S. Maragathasundari and S. Srinivasan, Analysis of $M / G / 1$ feedback queue with three stage multiple sever vacation, Applied Mathematical Sciences, 6 (2012), 62216240.

[15] S. Maragathasundari and S. Srinivasan, Analysis of $M / G / 1$ queue with triple stage of service having compulsory vacation and service interruptions, Far East Journal of Mathematical Sciences, 69 (2012), 61-80. 


\section{BATCH ARRIVAL QUEUE WITH TWO STAGES}

[16] S. Maragathasundari and S. Srinivasan, Three phase $M / G / 1$ queue with Bernoulli feedback and multiple server vacation and service interruptions, International Journal of Applied Mathematics and Statistics, 33.3 (2013), 55-70.

[17] S. Maragathasundari and S. Srinivasan, Analysis of $M / G / 1$ feedback queue with multi stage and multiple server vacation, International Conference on Mathematical Sciences and Computer Engineering, (ICMSCE 2012, MALAYSIA), 90-96.

[18] S. Maragathasundari and S. Srinivasan, Analysis of transient behaviour of $M / G / 1$ queue with single vacation, International Journal of Pure and Applied Mathematics, 76 (2012), 149-156.

[19] S. Maragathasundari and S. Srinivasan, Multi phase $M / G / 1$ queue with Bernoulli feedback and multiple server vacation, International Journal of Computer Applications, $\mathbf{5 2 . 1}$ (2012), 18-23.

[20] S. Maragathasundari and S. Srinivasan, Retrial queue with phase vacations, European Journal of Scientific Research, 93 (2012), 226-235.

[21] S. Maragathasundari and S. Srinivasan, Analysis of $M / M / 1$ queue for ATM facility, Global Journal of Theoretical and Applied Mathematics Sciences, 2 (2012), 41-46.

[22] S. Maragathasundari and S. Srinivasan, $M / M / C$ queueing model for waiting time of customers in Bank sectors, International Journal of Mathematical Sciences and Applications, 1.3, (2011), 1569-1575.

[23] S. Maragathasundari and S. Srinivasan, Time dependent solution of a non Markovian Queue with triple stage of service having compulsory vacation and service interruptions, International Journal of Computer Applications, 41.7 (2012), 37-43.

[24] S. Maragathasundari, S. Srinivasan, and A. Ranjitham, A batch arrival nonMarkovian queue with three types of service, International Journal of Computer Applications, 83.5 (2013), 43-47.

[25] S. Maragathasundari, S. Srinivasan, and A. Ranjitham (2014), Batch arrival queueing with two stages of service, Int. Journal of Math. Analysis, 8, 247-258.

[26] V. Thangaraj and S. Vanitha, Single server feedback queue with two types of service having general distribution, International Mathematical Forum, 5 (2010), 15-33.

MSC2010: 60K25, 60K30

Key words and phrases: batch arrival, Bernoulli schedule, random breakdown, steady state, queue size

Dept. of Maths, Velammal Institute of Technology, Chennai, India

E-mail address: maragatham01@gmail.com

Dept. of Maths, B. S. Abdur Rahman University, Chennai, India

E-mail address: srinivasan@bsauniv.ac.in

Dept. of Maths, Velammal Institute of Technology, Chennai, India

E-mail address: ranjithamsurya@yahoo.co.in 